\title{
Optical Parametric Oscillation in Dielectric Multipolar Nanostructures
}

\author{
Saman Jahani, Arkadev Roy, and Alireza Marandi \\ Department of Electrical Engineering, California Institute of Technology, CA, USA
}

\begin{abstract}
We present a theoretical framework using time-domain slowly-varying envelope approximation to study optical parametric oscillators (OPOs) in nanostructures with multipolar Mie resonances. We show feasibility of wavelength-scale OPOs which can be useful for numerous applications. @ 2020 The Author(s)
\end{abstract}

OPO is a nonlinear resonator for down-conversion of an input pump at the frequency of $\omega_{p}$ to signal and idler outputs at frequencies $\omega_{s}$, and $\omega_{i}$, such that $\omega_{p}=\omega_{s}+\omega_{i}$. In a degenerate OPO $\left(\omega_{s}=\omega_{i}=\omega_{p} / 2\right)$ operating below threshold the output signal can be a squeezed vacuum state ${ }^{1}$, while above threshold, the OPO illustrates binary phase states which can be considered as a spin in an Ising network ${ }^{2}$. Miniaturization of OPOs to nanoscale enables us to make efficient on-chip sources of quantum states as well as large-scale Ising networks ${ }^{3}$. Rapid progress in light confinement in all-dielectric nanostructures ${ }^{4,5}$ has raised the question about the possibility of miniaturization of OPOs into wavelength-scale. The light confinement in wavelength-scale resonators is based on localized multipolar Mie resonances. However, the existing theories have been developed for large-scale OPOs in which the pump and signals are considered as propagating waves passing through a cavity ${ }^{6}$. Hence, they cannot directly be applied to the wavelength-scale OPOs.
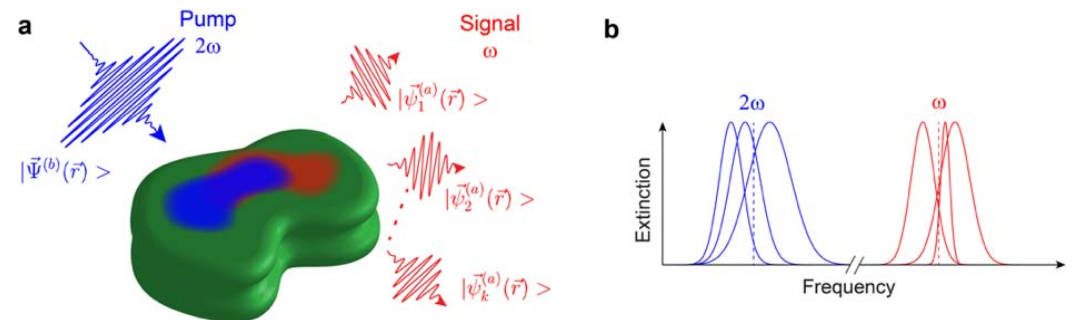

Fig. 1. (a) Arbitrarily shaped wavelength-scale optical parametric oscillator. The resonator supports normal modes at the pump frequency $(2 \omega)$ and signal frequency $(\omega)$. (b) The linear response of the resonator at the pump and signal frequencies. By knowing the eigen-frequencies of the modes and their quality factors as well as the overlap between the pump excitation and the signal modes, we can estimate the pump power threshold $\left(P_{t h}\right)$ for parametric oscillation.

Here, we propose a general theory to estimate the OPO threshold in multi-mode wavelength-scale resonators (Fig. 1). We expand the signal mode to the normal eigen-modes, and we approximate the nonlinear dynamic of the electric field with a slowly varying envelope evolving in timedomain. As a result, the wave equation for a degenerate OPO $\left(\omega_{s}=\omega_{p} / 2=\omega\right)$ is simplified to:

$$
\frac{d}{d t} a_{k}=\left(i \delta \omega_{k}-\frac{\alpha_{k}}{2}\right) a_{k}+\sum_{l} i b \eta_{l k} a_{l}^{*}
$$

where $a_{k}$ is the electric field amplitude of the $\mathrm{k}^{\text {th }}$ mode at signal frequency, $\alpha_{k}$ is the decay constant of the $\mathrm{k}^{\text {th }}$ cavity mode, $\delta \omega_{k}=\omega-\omega_{k}$ is the frequency detuning between the signal and the $\mathrm{k}^{\text {th }}$ mode, $b$ is the amplitude of the pump excitation, and $\eta_{l k} \propto\left\langle\psi_{l}^{(a) *}(r) \Psi^{(b)}(r) \psi_{k}^{(a)}(r)\right\rangle$ is the nonlinear coupling coefficient which is proportional to the overlap between the signal eigenmodes, $\left|\psi_{l, k}^{(a)}\right\rangle$, and the pump mode, $\left|\Psi^{(b)}\right\rangle$. The steady-state response of the above equation combined with its complex conjugate can be written in a matrix form: 
$\boldsymbol{\mathcal { M }}(b)\left[a_{1}, a_{1}^{*}, \cdots, a_{k}, a_{k}^{*}, \cdots\right]^{T}=0$. At threshold, the determinant of the matrix $\mathcal{M}$ must be zero to have non-trivial solution for the signal modes. The eigen-vector of $\mathcal{M}$ at threshold is the oscillating signal which is the superposition of the eigenmodes. Figure 2 illustrates an AlGaAs nanosphere as a wavelength-scale OPO. even with the pump peak power of tens of watts, it is possible to surpass the threshold as long as the signal frequency overlaps with the cavity multipolar frequencies.

a
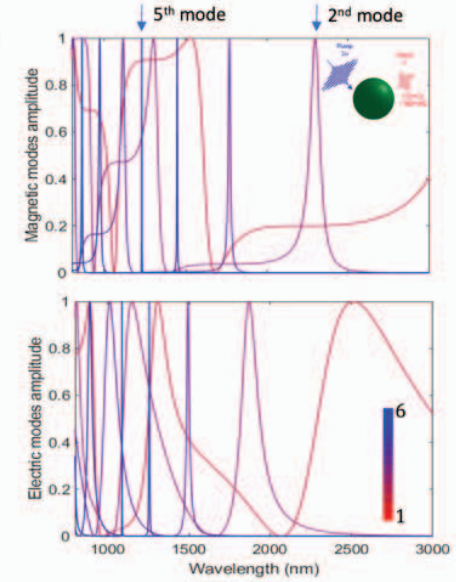

b

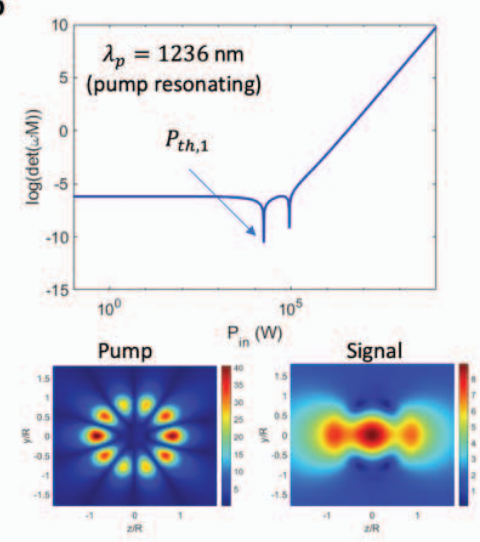

c

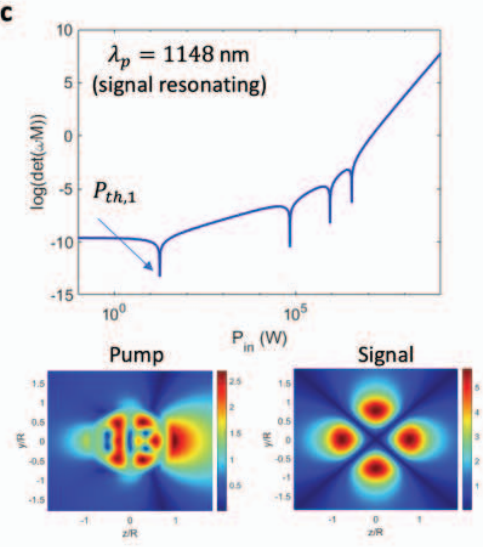

Fig. 2. OPO in a nanoscale dielectric sphere. (a) Scattering coefficients of electric and magnetic modes for an AlGaAs sphere with a radius of $400 \mathrm{~nm}$. (b) The pump frequency is set to excite the $5^{\text {th }}$ magnetic mode. The zeros of the determinant of the nonlinear coupled-mode matrix determines the OPO thresholds for supermodes. The electric field at pump frequency (left inset) is enhanced because of the resonance, but due the detuning for signal modes, the oscillation threshold $\left(P_{t h}\right)$ is high. The signal is the superposition of the electric dipole (ED), magnetic dipole (MD), electric quadrupole (EQ), and magnetic quadrupole (MQ) modes (right inset). The pump is an x-polarized plane wave propagating in the z-direction. The axes are normalized to the core radius. (c) The pump frequency is set such that the half harmonic is at MQ eigen-frequency (right inset). The pump is the superposition of multipolar modes (left inset). Since the signal is resonating at MD frequency, the threshold is significantly reduced.

Our approach is generalized to predict OPO in a wide range of nanostructured resonators, such as bound state in continuum ${ }^{7,8}$, photonic crystal $^{9}$, and inversely designed cavities ${ }^{10}$. Engineering the Mie resonances can lead to enhanced nonlinear cross couplings of the multi-mode OPOs which can significantly reduce the OPO threshold compared to single-mode OPOs. Our theoretical framework can be used to study non-degenerate OPOs. It can also establish a connection between up-conversion processes in nanostructures and parametric down-conversion. This allows us to explore the possibility of OPO in the existing structures which have been explored for sumfrequency/second-harmonic generation. Wavelength-scale OPOs can open unprecedented opportunities for classical and quantum nonlinear photonics.

\section{References:}

1. Wu, L.-A., Kimble, H. J., Hall, J. L. \& Wu, H. Phys. Rev. Lett. 57, 2520-2523 (1986).

2. Marandi, A., Wang, Z., Takata, K., Byer, R. L. \& Yamamoto, Y. Nat. Photonics 8, 937-942 (2014).

3. McMahon, P. L. et al. Science 354, 614-617 (2016).

4. Jahani, S. \& Jacob, Z. Nat. Nanotechnol. 11, 23-36 (2016).

5. Smirnova, D. \& Kivshar, Y. S. Optica 3, 1241-1255 (2016).

6. Schwob, C. et al. Appl. Phys. B 66, 685-699 (1998).

7. Carletti, L., Koshelev, K., De Angelis, C. \& Kivshar, Y. Phys. Rev. Lett. 121, 033903 (2018).

8. Marino, G. et al. Optica 6, 1416-1422 (2019).

9. Minkov, M., Gerace, D. \& Fan, S. Optica 6, 1039-1045 (2019).

10. Lin, Z., Liang, X., Lončar, M., Johnson, S. G. \& Rodriguez, A. W. Optica 3, 233-238 (2016). 